\title{
Roles of intracellular fibroblast growth factors in neural development and functions
}

\author{
ZHANG Xu ${ }^{1 *}$, BAO Lan ${ }^{2}$, YANG Liu ${ }^{1}$,WU QingFeng ${ }^{1} \&$ LI Shuai ${ }^{1}$ \\ ${ }^{1}$ Institute of Neuroscience and State Key Laboratory of Neuroscience, Shanghai Institutes for Biological Sciences, \\ Chinese Academy of Sciences, Shanghai 200031, China; \\ ${ }^{2}$ State Key Laboratory of Cell Biology, Institute of Biochemistry and Cell Biology, Shanghai Institutes for Biological Sciences, \\ Chinese Academy of Sciences, Shanghai 200031, China
}

Received October 10, 2012; accepted November 12, 2012

\begin{abstract}
Fibroblast growth factors (FGFs) can be classified as secretory (FGF1-10 and FGF15-23) or intracellular non-secretory forms (FGF11-14). Secretory forms of FGF and their receptors are best known for their regulatory roles in cell growth, differentiation and morphogenesis in the early stages of neural development. However, the functions of intracellular FGFs remain to be explored. FGF12 and FGF14 are found to interact with voltage-gated sodium channels, and regulate the channel activity in neurons. FGF13 is expressed in primary sensory neurons, and is colocalized with sodium channels at the nodes of Ranvier along the myelinated afferent fibers. FGF13 is also expressed in cerebral cortical neurons during the late developmental stage. A recent study showed that FGF13 is a microtubule-stabilizing protein required for regulating the neuronal development in the cerebral cortex. Thus, non-secretory forms of FGF appear to have important roles in the brain, and it would be interesting to further investigate the functions of intracellular FGFs in the nervous system and in neural diseases.
\end{abstract}

fibroblast growth factors, nervous system, development, microtubule, ion channel, X-linked mental retardation

Citation: Zhang X, Bao L, Yang L, et al. Roles of intracellular fibroblast growth factors in neural development and functions. Sci China Life Sci, 2012, 55: 1038-1044, doi: 10.1007/s11427-012-4412-x

Fibroblast growth factors (FGFs) belong to a family of signaling proteins that play diverse roles in cell growth, differentiation, morphogenesis and developmental processes [1-4]. Members of the FGF superfamily can be classified as secretory or intracellular non-secretory forms (Figure 1). Secretory FGFs (FGF1-10 and FGF15-23) contain an $\mathrm{N}$-terminal signal peptide for secretion and function via their receptors (FGF receptor 1-4), whereas intracellular FGFs (FGF11 family, FGF11-14) lack the signal peptide and thus may function intracellularly [5,6]. FGF11, FGF12, FGF13 and FGF14 were previously named FGF homologous factors (FHF) 3, FHF1, FHF2 and FHF4, respectively. These four FGF members are prominently expressed in the nervous system. They form a distinct family and show

*Corresponding author (email: xu.zhang@ion.ac.cn)
$30 \%-50 \%$ amino acid sequence identity with other FGFs [7]. Within these four FGFs, different members share 60\%-70\% amino acid sequence identity.

Each intracellular FGF gene has two or more sites for initiating transcription, which leads to the generation of multiple messenger RNAs with distinct first exons spliced to common exons II-V. These are then translated to produce proteins with different amino-terminal sequences [5] (Figure 1). Of the four intracellular forms of FGF, "A" isoforms contain long $\mathrm{N}$-terminal extensions that show extensive sequence homology to one another. "A" isoforms contain a nuclear localization signal (NLS) in the N-treminal region, whereas "B" isoforms do not have NLS. Thus, "A" isoforms transfected into cells often show nuclear localization, while " $\mathrm{B}$ " isoforms display cytoplasmic localization.

Accumulated evidence shows that secretory FGFs and 


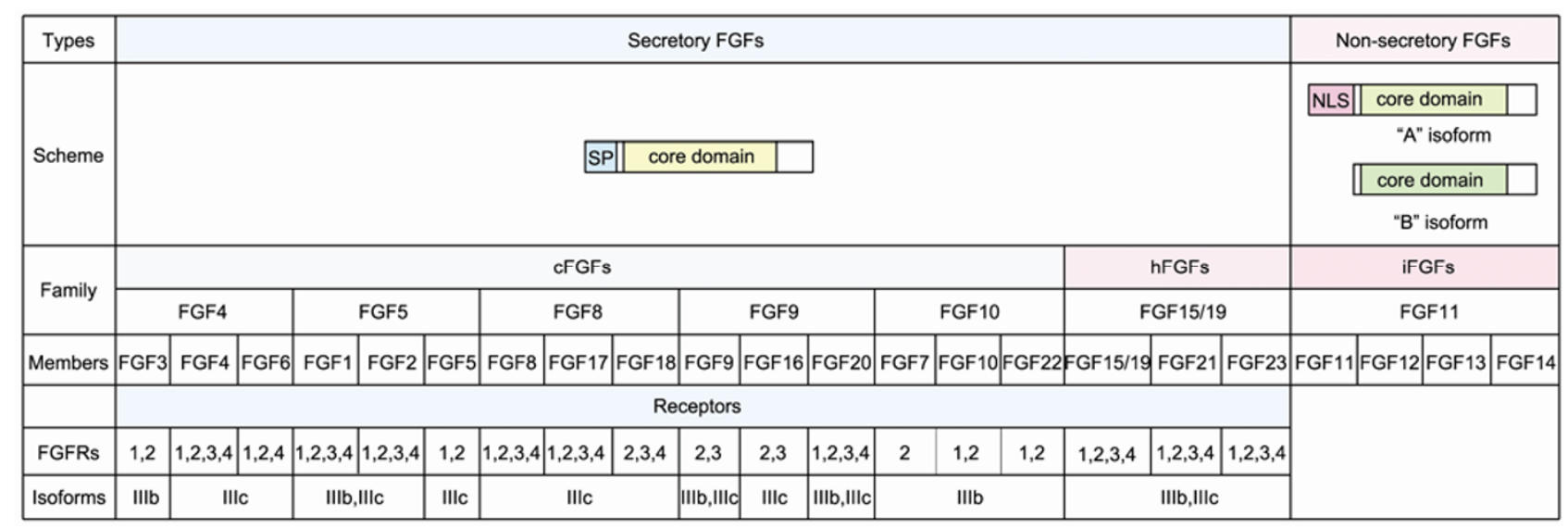

Figure 1 Family members of FGF ligands and receptors. Until now, 23 members (FGF1-FGF23) have been identified in humans and mice. The mammalian FGFs can be divided into the 7 families. Among them, FGF4, FGF5, FGF8, FGF9 and FGF10 families are defined as canonical FGFs (cFGFs), the FGF15/19 family is defined as hormone-like FGFs (hFGFs) and the FGF11 family is known as intracellular FGFs (iFGFs). All FGFs contain the homologous region of $\sim 120$ amino acids which is known as the core domain. Most FGFs have N-terminal signal peptides (SP) and can be secreted constitutively. However, the FGF9 family (FGF9, 16 and 20) have uncleavable signal peptides but can still be efficiently secreted. FGF1 and FGF2 lack signal peptides and can be released by exocytosis or from damaged cells which are non-canonical release pathways, independent of the endoplasmic reticulum-Golgi pathway. In particular, the FGF11 family (FGF11, 12, 13 and 14) which lacks signal peptides remains intracellular and functions within cells in a receptor-independent manner. FGF11 family genes have two or more isoforms produced by different transcription initiations. The A isoforms of FGF11 family members contain $\mathrm{N}$-terminal nuclear localization signals (NLSs). FGF receptors are composed of 4 members (FGFR1-4). The receptors can be further divided to IIIb and IIIc isoforms. The binding specificity of FGF ligands and receptors are shown in the figure.

their receptors play essential roles in the early stages of neural development [4]. Some secretory FGFs are also involved in affective disorders and modulate emotionality in animal models [8]. Conversely, knowledge about the functions and related mechanisms of intracellular FGFs in the nervous system is still limited when compared with what is known about secretory FGFs and their receptors. It is known that intracellular FGFs can interact with voltage-gated $\mathrm{Na}^{+}\left(\mathrm{Na}_{\mathrm{v}}\right)$ channels, and are required for normal motor function [5]. Recently, we also found that FGF13B is required for the development of the cerebral cortex [9].

Although the expression and functions of FGFs have been summarized and discussed recently [4,5], we feel that it is necessary to further summarize and discuss the research progress of intracellular FGFs in particular. Therefore, the present review will focus on recent studies of the expression and functional roles of intracellular FGFs in the nervous system (Table 1). The potential roles of intracellular FGFs in neural diseases will be also discussed.

\section{Expression of intracellular FGFs in the nervous system}

Members of the FGF11 family are expressed in cerebral cortical neurons during development $[5,7,9,21]$. FGF13 is also widely distributed in the developing brain with an expression level $\sim 5$-fold higher than FGF11, FGF12 and FGF14 [7]. Two alternatively spliced isoforms of FGF13 have been identified: FGF13A, which contains a NLS, and cytoplasmic FGF13B which does not contain a NLS [7,22].
FGF13B expression in the cerebral cortex of mice and rats gradually increases from embryonic day 14 (E14) until postnatal day 7 (P7), after which its expression level begins to decrease [9]. In the developing brain of mice, FGF13 is present in the subplate (SP), ganglionic eminences (GE) and proliferative zones of the cortical wall [the ventricular zone (VZ) and the subventricular zone (SVZ)] at E14. It is present in the cortical plate (CP) of the cerebral cortex, hippocampus and striatum from E17 to P14. FGF13 expression is largely absent in the adult mouse brain, except for low levels in the hippocampus.

In contrast to brain, the dorsal root ganglia (DRG) show expression of FGF13 and FGF14 in adult animals [23,24]. FGF13 is expressed in $60 \%$ of DRG neurons under normal circumstances [23]. It has been shown that the gene expression profiles of DRG and the dorsal spinal cord are modified by peripheral nerve injury, leading to gene-regulation of many molecules, such as neurotransmitter receptors, ion channels and the $\mathrm{Na}^{+}, \mathrm{K}^{+}$-ATPase activator [25-30]. Indeed, after sciatic nerve transection, the expression of FGF2 and FGF7 increased in the DRG, whereas FGF13 expression decreased $[23,24]$. Seven days after nerve injury, FGF13containing neurons decreased to $18 \%$, and partially recovered to $40 \%$ at 28 days after injury. Despite these observed changes in FGF expression, their functional role in DRG neurons under normal circumstances and after nerve injury remain to be explored.

It is interesting to note that the expression patterns of non-secretory FGFs in the central nervous system are different from that in the peripheral nervous system during development and adulthood. A particular example is the 
Table 1 Function of FGF11 family members in the nervous system

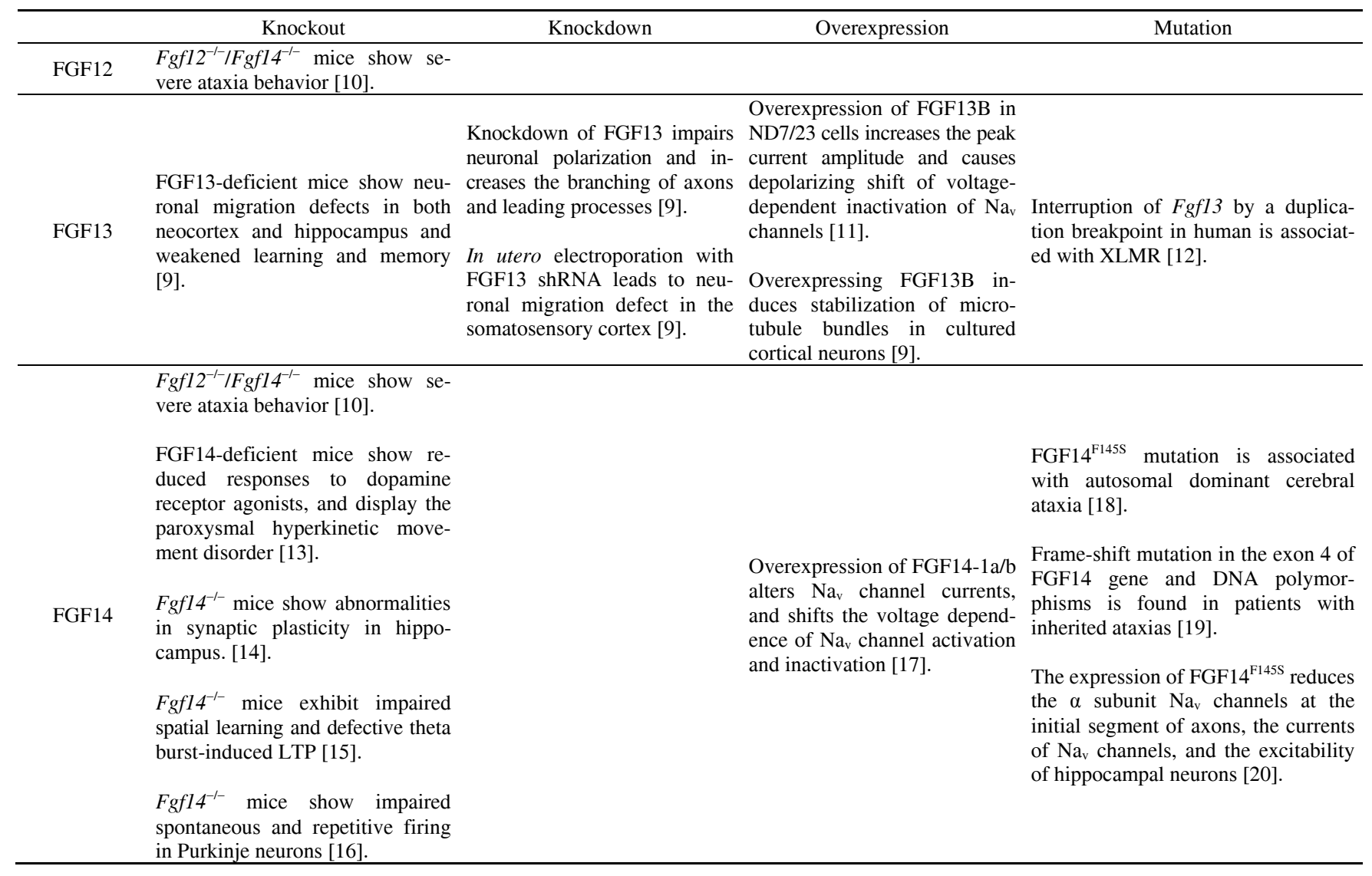

differential FGF13 expression pattern, where FGF13 is expressed in the brain during development but not in adulthood, whereas it is present in adult DRG neurons [23,24]. Such distinct expression patterns suggest differential roles of these FGFs in the nervous system throughout life.

\section{Role of intracellular FGFs in modulation of $\mathrm{Na}_{v}$ channel activity}

With regard to the function of intracellular FGFs, accumulated evidence shows that they are important for regulating the activity of $\mathrm{Na}_{v}$ channels (Table 1). Intracellular FGFs are often colocalized with $\mathrm{Na}_{\mathrm{v}}$ channels at the initial segments of axons and nodes of Ranvier [10,31,32]. Therefore, these FGFs might modulate the intiation and the propagation of action potentials [11,33-35]. FGF12B was found to bind to $\mathrm{Na}_{\mathrm{v}} 1.5$ and $\mathrm{Na}_{\mathrm{v}} 1.9$ channels, but not to $\mathrm{Na}_{\mathrm{v}} 1.7$ or $\mathrm{Na}_{\mathrm{v}} 1.8$ [36,37]. The interaction of FGF12B with $\mathrm{Na}_{\mathrm{v}} 1.5$ channel caused a hyperpolarizating shift in steady-state inactivation [37]. FGF13B was found to associate with $\mathrm{Na}_{\mathrm{v}} 1.6$, which is present at the nodes of Ranvier along central and peripheral axons and unmyelinated afferent fibers in the peripheral nervous system. FGF13B increased the amplitude of $\mathrm{Na}_{\mathrm{v}} 1.6$ sodium currents in a transfected DRG-derived cell line ND7/23 [10]. Two alternatively spliced N-terminal
FGF14 variants, FGF14-1a an FGF14-1b, differentially regulate currents produced by $\mathrm{Na}_{\mathrm{v}} 1.2$ and $\mathrm{Na}_{\mathrm{v}} 1.6$ channels [38]. Furthermore, intracellular FGF12 may be a component of tissue-specific protein kinase signaling modules, since FGF12 was found to interact with the MAP kinase (MAPK) scaffold protein Islet-Brain-2 (IB2) in the brain [22]. It remains unclear whether these intracellular FGFs increase the open probability of channels upon membrane depolarization by binding to the $\mathrm{C}$-terminus of $\mathrm{Na}_{\mathrm{v}}$ channels, by inserting more $\mathrm{Na}_{\mathrm{v}}$ channels into the plasma membrane from the cytosolic pool, or by recruiting a kinase to the channel complex and the subsequent phosphorylation of the channel. It would be interesting to study the correlation between the regulation of $\mathrm{Na}_{\mathrm{v}}$ channel activity and the signaling modules of intracellular FGFs in neurons.

Analysis of the crystal structure of FGF13A reveals a conserved surface required for binding to the $\mathrm{C}$-terminal domain of the pore-forming $(\alpha)$ subunits of $\mathrm{Na}_{\mathrm{v}}$ channels and for modulating the inactivation of $\mathrm{Na}_{\mathrm{v}}$ channels by partially occluding the inner face of the pore [17]. The 152-residue-long homologous region of intracellular FGFs is sufficient for channel binding, and the 18-residue-long, C-terminal extension of the homologous region plays an essential role in channel binding [17]. Alternative splicing at the N-terminus of intracellular FGFs does not contribute to their interaction with the C-terminal domain of $\mathrm{Na}_{\mathrm{v}}$ 
channels. FGF13A induces a greater shift in the voltage-dependence of inactivation when compared with FGF13B [10,17,39]. This suggests that the alternatively spliced N-terminus of FGF13A is more effective in occluding the channel pore.

Animal models of FGF-deficiency correlate with human diseases and give insight into the functions of intracellular FGFs. Studies show that $F g f 12$ knockout mice display muscle weakness [5] (Table 1). FGF14-deficient mice develop ataxia, paroxysmal hyperkinetic movement disorder and cognitive impairment $[13,32,40]$. This may be attributed to the loss of FGF14, which impairs the intrinsic excitability of cerebellar Purkinje neurons [15] and the long-term potentiation of synapses in the hippocampal CA1 region $[16,31,40]$. Likewise in humans, an autosomal dominant missense mutation, FGF14 ${ }^{\mathrm{F} 145 \mathrm{~S}}$, results in progressive spinocerebellar ataxia and cognitive impairment $[14,41]$. This is because the stability of FGF14 ${ }^{\mathrm{F} 145 \mathrm{~S}}$ is reduced, which leads to a loss of FGF14 function $[18,41]$. Furthermore, FGF14 ${ }^{\mathrm{F} 145 \mathrm{~S}}$ may disrupt the interaction of FGF14 with $\mathrm{Na}_{\mathrm{v}} 1.2$ channel and impair neuronal excitability [42]. Frameshift mutation and polymorphisms of $\mathrm{Fgfl} 4$ are also found in patients with inherited ataxias [20]. Therefore, FGF12 and FGF14 are important for regulating the neurotransmission of motor-functions. The functional roles of FGF13 expressed in adult hippocampal neurons and DRG neurons remain to be further investigated.

\section{FGF13 functions in neural development}

The extensive expression of FGF13 in the developing brain has prompted studies to investigate its functions. Early studies showed that FGF13 could induce tyrosine phosphorylation of mitogen-activated protein kinase (MAPK) and phospholipase C-gamma (PLC- $\gamma$ ) in hippocampal astrocytes [19]. Treatment of neuronal cultures from rat embryonic cortex with FGF13 increased the number of neurons containing gamma-aminobutyric acid (GABA) and choline acetyltransferase enzymes [19]. FGF13 is also involved in Xenopus neural differentiation [43]. In addition, FGF13 may be involved in limb development [44]. Pretreatment with intravenous FGF13 reduces infarct volume and ameliorates neurological deficits following focal cerebral ischemia in rats [45].

During brain development, cortical neurons migrate from the site of their last mitotic division towards their final destination and generate the proper neural circuits [46-48]. Proper regulation of microtubule dynamics during axon/leading process formation is crucial for the transition of neurons from the multipolar stage to a bipolar morphology, and is a prerequisite for initiating radial migration [49-51]. Tubulins, a number of microtubule-regulating proteins that promote microtubule assembly and proteins that protect microtubules from depolymerization (such as microtubule- stabilizing proteins), are all involved in this regulatory process [52]. Our recent study shows that FGF13B is a microtubule-stabilizing protein that is enriched in the growth cones of cortical neurons [9] (Table 1). FGF13 was found to promote microtubule polymerization and stabilization, enabling the polarization of developing cortical neurons. Knockdown of FGF13 expression impairs the polarization and migration of cortical neurons in developing brains of mice and rats [9] (Figure 2). Neuronal migration was delayed in both the neocortex and the CA1 region of hippocampus of Fgfl3 knockout mice. Learning and memory were also impaired in these FGF13-deficient mice, although the positioning of neurons in the neocortex and hippocampus of adult mutant mice was similar to that in control mice [9]. These data indicate an essential role of FGF13 in establishing neural circuits in the cerebral cortex and enabling cognitive functions.

The core function of FGF13B in brain development is its direct interaction with tubulin via a tubulin-binding domain, FGF13B ${ }^{\text {S104-Q111 }}$ [9]. Several members of the microtubulestabilizing proetein family, such as microtubule-associated proteins and doublecortin (DCX), are essential for the early phase of neuronal migration [53-57]. Reduction in DCX also attenuates the transition of multipolar neurons to bipolar ones in the developing cortex [54,58]. Interestingly, FGF13 is enriched in the axons of cortical neurons while its level in the dendrites is lower than DCX [9]. FGF13 and DCX may function independently during neural development, because the inhibition of neuronal migration caused by loss of DCX could be rescued by overexpressing FGF13 and vice versa. Therefore, it would be interesting to investigate the differential functions and correlations among FGF13, DCX and other microtubule-stabilizing proteins.

\section{FGF13 in X-linked mental retardation}

In humans, FGF11, FGF12, FGF13 and FGF14 genes are located on chromosome $17,3, \mathrm{X}$ and 13 , respectively [7]. $\mathrm{X}$-linked mental retardation (XLMR) is an inherited intellectual disability with disordered neural development arising from many mutations along the $\mathrm{X}$ chromosome. Although mutations resulting in XLMR have been reported in 102 genes [59-61], only a few of them have been proven to directly cause intellectual disability via defined mechanisms that are supported by experimental approaches, such as in animal models. Interestingly, FGF13 is a candidate gene for the syndromal and non-specific forms of XLMR mapped to the q26 region of the $X$ chromosome $[5,12,61,62,64]$. Direct evidence for an important role of FGF13 gene in XLMR is the impairment of learning and memory in FGF13-deficient mice [9]. We found that FGF13 loss delayed neuronal migration in both the neocortex and the hippocampus, and impaired learning and memory [9], indicating that the FGF13 function is essential for the development of cortical 

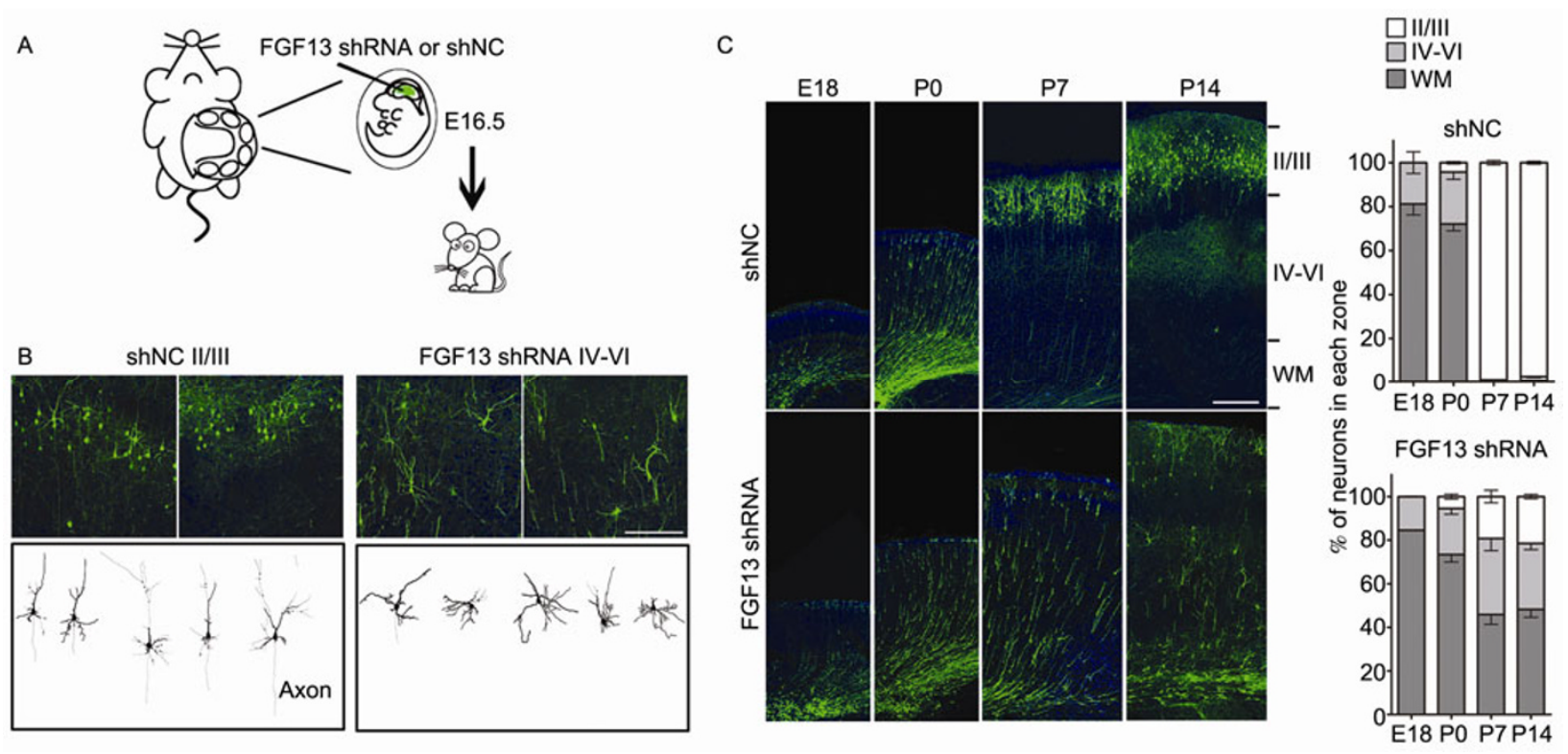

Figure 2 FGF13 is required for cerebral cortical development. A, To reduce the FGF13 expression, in utero electroporation with plasmids expressing short-hairpin-interfering RNA targeting FGF13 mRNA (FGF13 shRNA) or a negative control (shNC) was carried out at E16.5 in rats [9]. The brains were analyzed at different developmental stages. B, The FGF13 shRNA-expressing cortical neurons (green fluorescent protein-positive) examined at P14 showed multipolar instead of normal bipolar morphology. Scale bar, $100 \mu \mathrm{m}$. C, FGF13 knockdown results in a neuronal migration defect in the somatosensory cortex of rats. The radial migration of cortical neurons was delayed in the deep cortical layers. Scale bar, $200 \mu \mathrm{m}$.

structures and cognitive functions.

Duplication in Xq26 may contribute to XLMR [61,63,65]. Duplication may disrupt the coding or regulatory region of the gene located at its boundaries, or induce a dosage effect of proteins encoded by genes located within the duplicated region. In a Börjeson-Forssman-Lehmann syndrome-like patient, FGF13 may be interrupted by a duplication breakpoint [63]. However, it has to be pointed out that the phenotypes induced by FGF13 mutations can be different because these various mutations can generate diverse isoforms [44,63]. Different FGF13 isoforms or mutants may have distinct expression patterns, subcellular distribution and functions in development. Therefore, the phenotypes of FGF13 gene mutations may be determined largely by the mutation sites and the mutated products.

Although FGF13 is transiently expressed in the cerebral cortex only from E14 until P14 with a peak level at P7, FGF13-deficient mice exhibit marked defects in learning and memory. Therefore, the delayed neuronal migration and increased collateral branching of axons could disrupt the formation of the neural circuits and networks required for generating cognitive functions. At the moment, it remains unclear how synaptogenesis, action potential firing and synaptic transmission might be affected by loss of FGF13 function in adulthood, but some effects are expected because FGF13 may regulate neuronal excitability through modulating $\mathrm{Na}_{\mathrm{v}}$ channels [10,17,32]. Therefore, FGF13deficient mice may be a useful animal model for studying XLMR-related mechanisms.
This work was supported by the National Natural Science Foundation of China (Grant Nos. 31130066 and 30630029), National Basic Research Program of China (Grant Nos. 2011 CBA00400 and 2009CB522005), and Strategic Priority Research Program (B) of Chinese Academy of Sciences (Grant No. XDB02010200).

1 Burgess W H, Maciag T. The heparin-binding (fibroblast) growth factor family of proteins. Annu Rev Biochem, 1989, 58: 575-606

2 Rifkin D B, Moscatelli D. Recent developments in the cell biology of basic fibroblast growth factor. J Cell Biol, 1989, 109: 1-6

3 Yamaguchi T P, Rossant J. Fibroblast growth factors in mammalian development. Curr Opin Genet Dev, 1995, 5: 485-91

4 Guillemot F, Zimmer C. From cradle to grave: the multiple roles of fibroblast growth factors in neural development. Neuron, 2011, 71: 574-588

5 Goldfarb M. Fibroblast growth factor homologous factors: evolution, structure, and function. Cytokine Growth Factor Rev, 2005, 16: $215-220$

6 Itoh N, Ornitz D M. Functional evolutionary history of the mouse Fgf gene family. Dev Dyn, 2008, 237: 18-27

7 Smallwood P M, Munoz-Sanjuan I, Tong P, et al. Fibroblast growth factor (FGF) homologous factors: new members of the FGF family implicated in nervous system development. Proc Natl Acad Sci USA, 1996, 93: 9850-9857

8 Turner C A, Watson S J, Akil H. The fibroblast growth factor family: neuromodulation of affective behavior. Neuron, 2012, 76: 160-174

9 Wu Q F, Yang L, Li S, et al. Fibroblast growth factor 13 is a microtubule-stabilizing protein regulating neuronal polarization and migration. Cell, 2012, 149: 1549-1564

10 Wittmack E K, Rush A M, Craner M J, et al. Fibroblast growth factor homologous factor $2 \mathrm{~B}$ : association with $\mathrm{Na}_{\mathrm{v}} 1.6$ and selective colocalization at nodes of Ranvier of dorsal root axons. J Neurosci, 2004, 24: 6765-6775

11 Kole M H, Stuart G J. Signal processing in the axon initial segment. Neuron, 2012, 73: 235-247

12 Gedeon A K, Glass I A, Connor J M, et al. Genetic localisation of 
MRX27 to Xq24-26 defines another discrete gene for non-specific X-linked mental retardation. Am J Med Genet, 1996, 64: 121-124

13 Wozniak D F, Xiao M L, Xu L, et al. Impaired spatial learning and defective theta burst induced LTP in mice lacking fibroblast growth factor 14. Neurobiol Dis, 2007, 26: 14-26

14 Brusse E, de Koning I, Maat-Kievit A, et al. Spinocerebellar ataxia associated with a mutation in the fibroblast growth factor 14 gene (SCA27): A new phenotype. Mov Disord, 2006, 21: 396-401

15 Shakkottai VG, Xiao M, Xu L, et al. FGF14 regulates the intrinsic excitability of cerebellar Purkinje neurons. Neurobiol Dis, 2009, 33: 81-88

16 Xiao M, Xu L, Laezza F, et al. Impaired hippocampal synaptic transmission and plasticity in mice lacking fibroblast growth factor 14. Mol Cell Neurosci, 2007, 34: 366-377

17 Goetz R, Dover K, Laezza F, et al. Crystal structure of a fibroblast growth factor homologous factor (FHF) defines a conserved surface on FHFs for binding and modulation of voltage-gated sodium channels. J Biol Chem, 2009, 284: 17883-17896

18 Olsen S K, Garbi M, Zampieri N, et al. Fibroblast growth factor (FGF) homologous factors share structural but not functional homology with FGFs. J Biol Chem, 2003, 278: 34226-34236

19 Greene J M, Li Y L, Yourey P A, et al. Identification and characterization of a novel member of the fibroblast growth factor family. Eur $\mathbf{J}$ Neurosci, 1998, 10: 1911-1925

20 Dalski A, Atici J, Kreuz F R, et al. Mutation analysis in the fibroblast growth factor 14 gene: frameshift mutation and polymorphisms in patients with inherited ataxias. Eur J Hum Genet, 2005, 13: 118-120

21 Hartung H, Feldman B, Lovec H, et al. Murine FGF-12 and FGF-13: expression in embryonic nervous system, connective tissue and heart. Mech Dev, 1997, 64: 31-39

22 Schoorlemmer J, Goldfarb M. Fibroblast growth factor homologous factors are intracellular signaling proteins. Curr Biol, 2001, 11: 793-797

23 Li G D, Wo Y, Zhong M F, et al. Expression of fibroblast growth factors in rat dorsal root ganglion neurons and regulation after peripheral nerve injury. Neuroreport, 2002, 13: 1903-1907

24 Xiao H S, Huang Q H, Zhang F X, et al. Identification of gene expression profile of dorsal root ganglion in the rat peripheral axotomy model of neuropathic pain. Proc Natl Acad Sci USA, 2002, 99: 83608365

25 Zhang X, Xiao H S. Gene array analysis to determine the components of neuropathic pain signaling. Curr Opin Mol Ther, 2005, 7: 532537

26 Li K C, Zhang F X, Li C L, et al. Follistatin-like 1 suppresses sensory afferent transmission by activating $\mathrm{Na}^{+}, \mathrm{K}^{+}$-ATPase. Neuron, 2011, 69: 974-987

27 Li K C, Wang F, Zhong Y Q, et al. Reduction of follistatin-like 1 in primary afferent neurons contributes to neuropathic pain hypersensitivity. Cell Res, 2011, 21: 697-699

28 Zhang X. Pain research in China. Sci China Life Sci, 2010, 53: 356-362

29 Hucho T, Levine J D. Signaling pathways in sensitization: toward a nociceptor cell biology. Neuron, 2007, 55: 365-376

30 Campbell J N, Meyer R A. Mechanisms of neuropathic pain. Neuron, 2006, 52: 77-92

31 Lou J Y, Laezza F, Gerber B R, et al. Fibroblast growth factor 14 is an intracellular modulator of voltage-gated sodium channels. J Physiol, 2005, 569: 179-193

32 Goldfarb M, Schoorlemmer J, Williams A, et al. Fibroblast growth factor homologous factors control neuronal excitability through modulation of voltage-gated sodium channels. Neuron, 2007, 55: 449-463

33 Thaxton C, Pillai A M, Pribisko A L, et al. Nodes of Ranvier act as barriers to restrict invasion of flanking paranodal domains in myelinated axons. Neuron, 2011, 69: 244-257

34 Debanne D. The nodal origin of intrinsic bursting. Neuron, 2011, 71: 569-570

35 Kole M H. First node of Ranvier facilitates high-frequency burst encoding. Neuron, 2011, 71: 671-682

36 Liu C, Dib-Hajj S D, Waxman S G. Fibroblast growth factor homol- ogous factor 1B binds to the $\mathrm{C}$ terminus of the tetrodotoxin-resistant sodium channel $\mathrm{rNa}_{\mathrm{v}} 1.9 \mathrm{a}(\mathrm{NaN})$. J Biol Chem, 2001, 276: 1892518933

37 Liu CJ, Dib-Hajj S D, Renganathan M, et al. Modulation of the cardiac sodium channel $\mathrm{Na}_{\mathrm{v}} 1.5$ by fibroblast growth factor homologous factor 1B. J Biol Chem, 2003, 278: 1029-1036

38 Laezza F, Lampert A, Kozel M A, et al. FGF14 N-terminal splice variants differentially modulate $\mathrm{Na}_{\mathrm{v}} 1.2$ and $\mathrm{Na}_{\mathrm{v}} 1.6$-encoded sodium channels. Mol Cell Neurosci, 2009, 42: 90-101

39 Rush A M, Wittmack E K, Tyrrell L, et al. Differential modulation of sodium channel $\mathrm{Na}_{\mathrm{v}} 1.6$ by two members of the fibroblast growth factor homologous factor 2 subfamily. Eur J Neurosci, 2006, 23: 2551-2562

40 Wang Q, Bardgett M E, Wong M, et al. Ataxia and paroxysmal dyskinesia in mice lacking axonally transported FGF14. Neuron, 2002, 35: 25-38

41 van Swieten J C, Brusse E, de Graaf B M, et al. A mutation in the fibroblast growth factor 14 gene is associated with autosomal dominant cerebellar ataxia. Am J Hum Genet, 2003, 72: 191-199

42 Laezza F, Gerber B R, Lou J Y, et al. The FGF14 ${ }^{\mathrm{F} 145 \mathrm{~S}}$ mutation disrupts the interaction of FGF14 with voltage-gated $\mathrm{Na}^{+}$channels and impairs neuronal excitability. J Neurosci, 2007, 27: 12033-12044

43 Nishimoto S, Nishida E. Fibroblast growth factor 13 is essential for neural differentiation in Xenopus early embryonic development. J Biol Chem, 2007, 282: 24255-24261

44 Munoz-Sanjuan I, Simandl B K, Fallon J F, et al. Expression of chicken fibroblast growth factor homologous factor (FHF)-1 and of differentially spliced isoforms of FHF-2 during development and involvement of FHF-2 in chicken limb development. Development, 1999, 126: 409-421

45 Yao D L, Masonic K, Petullo D, et al. Pretreatment with intravenous FGF-13 reduces infarct volume and ameliorates neurological deficits following focal cerebral ischemia in rats. Brain Res, 1999, 818: 140146

46 Ayala R, Shu T, Tsai L H. Trekking across the brain: the journey of neuronal migration. Cell, 2007, 128: 29-43

47 Marin O, Valiente M, Ge X, et al. Guiding neuronal cell migrations. Cold Spring Harb Perspect Biol, 2010, 2: a001834

48 Yuan X. Axon guidance and neuronal migration research in China. Sci China Life Sci, 2010, 53: 304-314

49 Jaglin X H, Chelly J. Tubulin-related cortical dysgeneses: microtubule dysfunction underlying neuronal migration defects. Trends Genet, 2009, 25: 555-566

50 Witte H, Neukirchen D, Bradke F. Microtubule stabilization specifies initial neuronal polarization. J Cell Biol, 2008, 180: 619-632

51 Li L, Wei D, Wang Q, et al. MEC-17 deficiency leads to reduced $\alpha$-tubulin acetylation and impaired migration of cortical neurons. $\mathrm{J}$ Neurosci, 2012, 32: 12673-12683

52 Poulain F E, Sobel A. The microtubule network and neuronal morphogenesis: Dynamic and coordinated orchestration through multiple players. Mol Cell Neurosci, 2010, 43: 15-32

53 Teng J, Takei Y, Harada A, et al. Synergistic effects of MAP2 and MAP1B knockout in neuronal migration, dendritic outgrowth, and microtubule organization. J Cell Biol, 2001, 155: 65-76

54 Bai J, Ramos R L, Ackman J B, et al. RNAi reveals doublecortin is required for radial migration in rat neocortex. Nat Neurosci, 2003, 6: 1277-1283

55 Kappeler C, Saillour Y, Baudoin J P, et al. Branching and nucleokinesis defects in migrating interneurons derived from doublecortin knockout mice. Hum Mol Genet, 2006, 15: 1387-1400

56 Bouquet C, Soares S, von Boxberg Y, et al. Microtubule-associated protein $1 \mathrm{~B}$ controls directionality of growth cone migration and axonal branching in regeneration of adult dorsal root ganglia neurons. J Neurosci, 2004, 24: 7204-7213

57 Takei Y, Teng J, Harada A, et al. Defects in axonal elongation and neuronal migration in mice with disrupted tau and maplb genes. $\mathrm{J}$ Cell Biol, 2000, 150: 989-1000

58 Koizumi H, Higginbotham H, Poon T, et al. Doublecortin maintains bipolar shape and nuclear translocation during migration in the adult 
forebrain. Nat Neurosci, 2006, 9: 779-786

59 Lubs H A, Stevenson R E, Schwartz C E. Fragile X and X-linked intellectual disability: four decades of discovery. Am J Hum Genet, 2012, 90: 579-590

60 Tarpey P S, Smith R, Pleasance E, et al. A systematic, large-scale resequencing screen of $\mathrm{X}$-chromosome coding exons in mental retardation. Nat Genet, 2009, 41: 535-543

61 Gecz J, Shoubridge C, Corbett M. The genetic landscape of intellectual disability arising from chromosome X. Trends Genet, 2009, 25: 308-316

62 Malmgren H, Sundvall M, Dahl N, et al. Linkage mapping of a severe X-linked mental retardation syndrome. Am J Hum Genet, 1993,
52: 1046-1052

63 Gecz J, Baker E, Donnelly A, et al. Fibroblast growth factor homologous factor 2 (FHF2): gene structure, expression and mapping to the Borjeson-Forssman-Lehmann syndrome region in Xq26 delineated by a duplication breakpoint in a BFLS-like patient. Hum Genet, 1999, 104: 56-63

64 Shiloh Y, Litvak G, Ziv Y, et al. Genetic mapping of X-linked albinism-deafness syndrome (ADFN) to Xq26.3-q27.I. Am J Hum Genet, 1990, 47: 20-27

65 Solomon N M, Nouri S, Warne G L, et al. Increased gene dosage at $\mathrm{Xq} 26-\mathrm{q} 27$ is associated with X-linked hypopituitarism. Genomics, 2002, 79: 553-559

Open Access This article is distributed under the terms of the Creative Commons Attribution License which permits any use, distribution, and reproduction in any medium, provided the original author(s) and source are credited. 